\title{
Erythromycin Induced Nightmares
}

TO THE EDITOR: The word nightmare originates from the 13th century and is composed of night and mare, suggestive of an evil female spirit, afflicting sleepers with a feeling of suffocation, as they ride on their chests. Classified as frightening dreams that awaken the sleeper from rapid eye movement sleep, ${ }^{1}$ nightmares impact sleep quality and are linked to various health problems. Likewise, nightmares are comorbid to psychiatric condition. ${ }^{1,2}$

Erythromycin is a macrolide antibiotic. Erythromycin also has prokinetic properties, thus, it increases gastrointestinal motility via motilin receptor agonism. ${ }^{3}$ This case report presents a rare case of erythromycin-induced nightmares (Figure).

A 19-year-old lady with Ehlers-Danlos syndrome (hypermobility type) and delayed gastric emptying was administered $250 \mathrm{mg}$ erythromycin 4 times a day to increase gastrointestinal motility. The delayed gastric emptying was diagnosed on the ${ }^{13} \mathrm{C}$-octanoic-acid breath test. Within the first week after administration, the patient presented with nightmares, characterised as vivid dreams 3-7 nights a week. Preceding administration, this patient had never com-

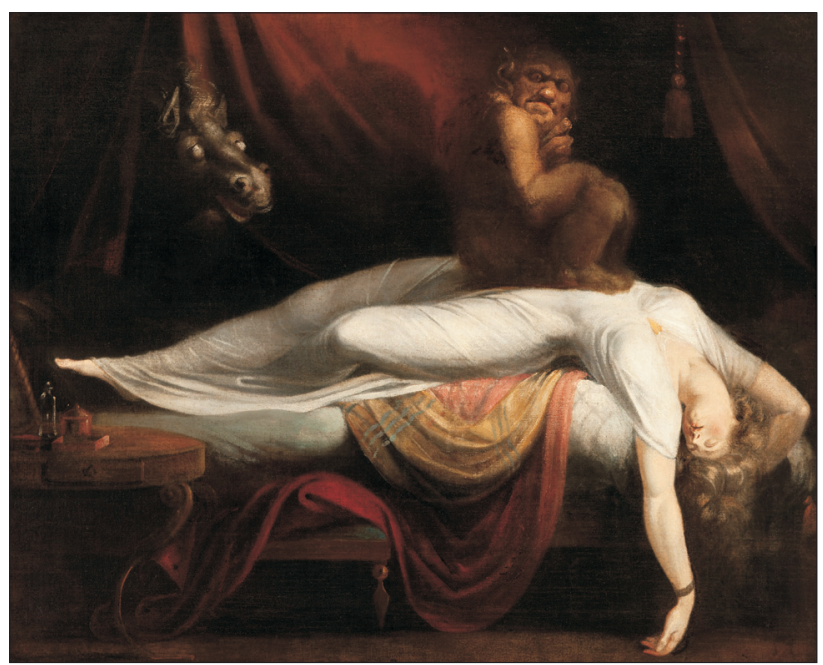

Figure. "The Nightmare", Henry Fuseli, 1789, oil on canvas. With permission from Detroit Institute of Arts. plained about debilitating nightmares and had no history of psychiatric disorders. Erythromycin was beneficial for her gastrointestinal symptoms and accordingly the frequency of nausea, regurgitation, and vomiting decreased. The patient found the nightmares disturbing and stopped the treatment for 3 weeks. The nightmares went into remission after a few days. However, as a consequence of discontinuation, the patients' gastrointestinal symptoms flared-up, and therefore she restarted the treatment with erythromycin, resulting in reappearance of nightmares within days. At the next appointment at a tertiary neurogastroenterology clinic, erythromycin was substituted for azithromycin $250 \mathrm{mg}$ on alternate days after a 1-week washout-phase. Subsequent follow-up visits have revealed no nightmares. Furthermore, the remission of the comorbid gastrointestinal symptoms has continued.

Only one previous case of erythromycin-induced nightmares exists. ${ }^{4}$ In that case, a young woman was administrated erythromycin $250 \mathrm{mg}$ a day to treat acne. Post-administration she reported nightmares, on average bi-weekly. In agreement with the present case, the probands are young females with debilitating nightmares and no medical history of psychiatric disorders that remit after cessation of erythromycin.

Concomitant medication is imperative for consideration. Besides erythromycin, the patient was only taking Gedarel and Movicol regularly. Depression is a well-recognized side-effect of contraceptives, and nightmares are correlated to depression. ${ }^{2,5}$ Regardless, Gedarel, as a cause of nightmares in this case is very unlikely, as the patient was using Gedarel for 2-3 years before administration of erythromycin. Furthermore, no drug interaction between Gedarel and erythromycin has been reported in the literature.

As cessation of erythromycin and remission of nightmares correspond, correlation is most plausible. No class-effect of macrolides has been established though clarithromycin and azithromycin have been reported to induce hallucinations, no reports of nightmares are available. ${ }^{6,7}$

A variety of medications may induce nightmares. Receptor modulation of the central nervous system is likely a mechanism of 
action. Medications like norepinephrine, serotonin, and dopamine, are associated with nightmares, albeit erythromycin has not been reported to interact with neurotransmitters. Other medications also under suspicion of inducing nightmares are gamma-aminobutyric-acid, acetylcholine, histamine, antiepileptics, antipsychotics, and some anesthetics (eg, propofol, thiopental, isoflurane, and ketamine). ${ }^{8}$ Statins, an inhibitor of the hydroxy-3-methyl-glutarylcoenzyme-A (HMG-CoA) reductase, are known to induce nightmares. By inhibiting HMG-CoA reductase, the de-novo synthesis of cholesterol is inhibited. This may lead to a reduction in serum cholesterol. Studies have identified low serum cholesterol as a potentially cause psychiatric condition (eg, sleep disturbances). ${ }^{9,10}$

This case signifies erythromycin as a potential cause of nightmares and an important correlation to consider in patients with refractory nightmares during erythromycin treatment. The gap of 3 decades between cases of erythromycin-induced nightmares denotes that the phenomenon is rare and/or underreported, as erythromycin is widely used. Further studies are warranted to examine the underlying mechanisms.

Maria E Møller, Qasim Aziz, and Jacob Juel

Center for Digestive Diseases, Blizard Institute of Cell and Molecular Science, Wingate Institute of Neurogastroenterology, Barts and the London School of Medicine and Dentistry, Queen Mary University of London, London, UK

1. American Academy of Sleep Medicine. The international classification of sleep disorders, 2001. Available from: http://www.esst.org/adds/ICSD. pdf (accessed 27 April 2016).

2. Schredl M, Reinhard I. Gender differences in nightmare frequency: a meta-analysis. Sleep Med Rev 2011;15:115-121.

3. Larson JM, Tavakkoli A, Drane WE, Toskes PP, Moshiree B. Advantages of azithromycin over erythromycin in improving the gastric emptying half-time in adult patients with gastroparesis. J Neurogastroenterol Motil 2010;16:407-413.

4. Black RJ, Dawson TA. Drug points. Erythromycin and nightmares. Br Med J (Chin Res Ed) 1988;296:1070.

5. Poromaa IS, Segebladh B. Adverse mood symptoms with oral contraceptives. Acta Obstet Gynecol Scand 2012;91:420-427.

6. Erkek N, Senel S, Karacan C. Visual hallucinations possibly associated with clarithromycin administration at therapeutic dosage in two children. Med Princ Pract 2009;18:332-334.

7. Schiff E, May K, Goldstein LH. Neuropsychiatric manifestations associated with azithromycin in two brothers. Eur J Clin Pharmacol 2010;66:1273-1275.

8. Pagel JF, Helfter P. Drug induced nightmares--an etiology based review. Hum Psychopharmacol 2003;18:59-67.

9. Tuccori M, Montagnani S, Mantarro S, et al. Neuropsychiatric adverse events associated with statins: epidemiology, pathophysiology, prevention and management. CNS Drugs 2014;28:249-272.

10. Agargun MY, Gulec M, Cilli AS, et al. Nightmares and serum cholesterol level: a preliminary report. Can J Psychiatry 2005;50:361-364.

\section{Conflicts of interest: None.}

Author contributions: Maria E Møller: drafting manuscript; Qasim Aziz: critical revision of manuscript for important intellectual content and clinical supervision; and Jacob Juel: drafting manuscript and revision of it critically for important intellectual content, and clinical revision and follow-up of patient. 